\title{
Correlates and genetics of self-reported sleep and awake bruxism in a nationwide twin cohort
}

\author{
Jari Ahlberg ${ }^{1}$ (D) | Maarit Piirtola ${ }^{2}$ | Frank Lobbezoo ${ }^{3}$ (D) | Daniele Manfredini ${ }^{4}$ (D) | \\ Tellervo Korhonen $^{2}$ | Ghizlane Aarab ${ }^{3}$ (D) | Christer Hublin ${ }^{5,6}$ | Jaakko Kaprio ${ }^{2,6}$
}

\begin{abstract}
${ }^{1}$ Department of Oral and Maxillofacial Diseases, University of Helsinki, Helsinki, Finland

${ }^{2}$ Institute for Molecular Medicine Finland FIMM, HiLIFE, University of Helsinki, Helsinki, Finland

${ }^{3}$ Department of Orofacial pain and Dysfunction, Academic Centre for Dentistry Amsterdam (ACTA), University of Amsterdam and Vrije Universiteit Amsterdam, Amsterdam, The Netherlands

${ }^{4}$ School of Dentistry, Department of Biomedical Technologies, University of Siena, Siena, Italy

${ }^{5}$ Finnish Institute of Occupational Health, Helsinki, Finland

${ }^{6}$ Department of Public Health, Faculty of Medicine, University of Helsinki, Helsinki, Finland
\end{abstract}

\section{Correspondence}

Jari Ahlberg, Department of Oral and Maxillofacial Diseases, University of Helsinki, Helsinki, Finland.

Email: jari.ahlberg@helsinki.fi

\begin{abstract}
Background: Sleep bruxism (SB) and awake bruxism (AB) have been considered different entities, although co-occurrence between them has been shown. While genetic factors have a marked influence on phenotypic variance in liability to $S B$, this remains unclear for $A B$.
\end{abstract}

Aim: To examine the degree of co-occurrence of $S B$ and $A B$, and whether they have common correlates and also twin similarity of $\mathrm{SB}$ and $\mathrm{AB}$ bruxism traits by zygosity and sex.

Methods: A questionnaire was mailed to all twins born 1945-1957 in Finland in 2012 ( $n=11766$ ). Age and sex adjusted logistic regression models were used. Twin similarity was assessed using polychoric correlations, and crosstwin-crosstrait correlations were computed.

Results: The response rate was $72 \%(n=8410)$. Any SB was reported by $14.8 \%$ and $\geq 3$ nights weekly by $5.0 \%$. Percentages for any $A B$ were $18.4 \%$ and $6.3 \%$, respectively. There was substantial co-occurrence $(29.5 \%)$ between $S B$ and $A B$, and several shared correlates were found. For $\mathrm{SB}$, the polychoric intra-class correlation was 0.366 in monozygotic (MZ) and 0.200 in dizygotic (DZ) pairs, without gender difference. A twofold crosstwin-crosstrait correlation was observed in MZ twins compared to DZ twins.

Conclusions: The risk factor profiles of $\mathrm{SB}$ and $\mathrm{AB}$ were largely but not entirely similar. The higher correlation in $M Z$ than in $D Z$ pairs suggests the influence of genetic factors on both $S B$ and $A B$. The higher crosstwin-crosstrait correlation in $M Z$ than in $D Z$ pairs suggests some degree of genetic influences shared by $S B$ and $A B$.

\section{KEYWORDS}

awake bruxism, genetic epidemiology, sleep bruxism, twins

\section{1 | INTRODUCTION}

Lobbezoo et al ${ }^{1}$ defined bruxism as a repetitive jaw-muscle activity characterised by clenching or grinding of the teeth and/or 
grading system of "possible," "probable" and "definite" SB or AB, the grade depending on the method used to assess bruxism. Since publication of this widely cited international consensus paper, its inclusion in both fourth and fifth editions of the Guidelines for Assessment, Diagnosis and Management of Orofacial Pain of the American Academy of Orofacial Pain, ${ }^{2,3}$ as well as in the third edition of the International Classification of Sleep Disorders, ${ }^{4}$ serves to confirm its rapid and broad adoption in the field. Recently, the consensus definition was revisited and updated by Lobbezoo et al as follows ${ }^{5}$ :

1. Sleep bruxism (SB) is a masticatory muscle activity during sleep that is characterised as rhythmic (phasic) or non-rhythmic (tonic) and is not a movement disorder or sleep disorder in otherwise healthy individuals.

2. Awake bruxism (AB) is a masticatory muscle activity during wakefulness that is characterised by repetitive or sustained tooth contact and/or by bracing or thrusting of the mandible and is not a movement disorder in otherwise healthy individuals.

In addition, it was noted that both definitions begin with "masticatory muscle activity," a phrase intended to emphasise the role of the masticatory muscles during sleep and wakefulness, viz., muscle work as the source of potential positive or negative consequences. ${ }^{6}$

During the past two decades, "definite" SB has been diagnosed by means of polysomnography performed in sleep laboratories, or data have been gathered by ambulatory devices in the home environment. According to large epidemiological studies, "possible" SB is considered to be present in about $6 \%-8 \%$ in adult populations, with no significant gender difference and a slight decrease with age, ${ }^{7,8}$ and half of the variation across populations has been suggested to be genetic in origin. ${ }^{9-11}$ Also, SB reportedly has several underlying factors: psychological (eg dissatisfaction, stress sensitivity and anxiety), ${ }^{12-14}$ behavioural and pharmacological (eg psychoactive medications, alcohol, tobacco and caffeine use), ${ }^{15,16}$ and physiological (eg sleep disordered breathing and reflux disease). ${ }^{17,18}$ These factors, with varying degrees of evidence for their role at present, are likely to act on an underlying genetic predisposition that is probably polygenic in nature given the lack of evidence for major genes, not to mention the lack overall of sufficiently powered genetic studies. Everyone has some degree of genetic predisposition and of triggering exposures, the relative mix varying for each patient. Yet, despite the many faces of SB, the problems involved in diagnosing it and the fact that it cannot be clearly linked to any pathophysiology or physiopathology, ${ }^{19}$ it is commonly agreed that SB mostly manifests during light sleep and towards arousal.

$A B$ in turn, with a reported prevalence up to $30 \%$ among adult populations, ${ }^{20}$ has mainly been assessed by self-report, together with some specific clinical signs in the oral cavity (eg tongue scalloping, linea alba on buccal mucosa). Whereas SB appears to have a time-variant nature, ${ }^{21}$ both that and the aetiology of $A B$ have remained unclear. Therefore, in addition to the information derived from a single dental visit, patients are asked to keep a diary for 1-2 weeks to report their masticatory muscle activity (viz., teeth clenching, mandible bracing/thrusting even without tooth contact). For this purpose, a smart phone app with a so-called ecological momentary assessment function was recently introduced. ${ }^{22-24}$ Based on repeated random alerts during wakefulness, the app seems promising for the study of real-time $A B$ and its correlates on a larger scale, but may also serve as a possible tool for ecological momentary intervention in the management of $A B$ by individuals. ${ }^{24}$

Even though $\mathrm{SB}$ and $\mathrm{AB}$ have commonly been considered as distinctly different entities, co-occurrence up to $20 \%$ has been reported. ${ }^{25}$ In order to clarify this issue, we aimed, as part of a nationwide study among older Finnish twins, to examine whether self-reported SB and AB overlapped within individuals, and whether they had shared correlates. An additional goal was to analyse twin similarity by computing polychoric correlations of $S B$ and $A B$ bruxism traits by zygosity (monozygotic (MZ) and dizygotic (DZ) pairs) and sex. We also computed crosstwin-crosstrait correlations to explore genetic influences shared by SB and $A B$.

\section{2 | MATERIAL AND METHODS}

In the Finnish Twin Cohort (17), a fourth wave of data collection by postal questionnaire was conducted in 2011-2012. The questionnaire was mailed to all twins living in Finland who had been in the cohort in 1975, irrespective of response to earlier questionnaires. Of 11766 twins born 1945-1957, 8410 (3753 men, 4657 women) returned the completed questionnaire. The response rate was $72 \%,{ }^{26}$ with mean age 60 years (range 53-67).

The domains examined covered multiple areas of health and risk factors (eg blood pressure and cholesterol measurements, diabetes, breathlessness, back pain, chronic diseases, use of common medications). Questions on sleep and bruxism (see below) were included. We derived smoking status based on detailed smoking history, and there were also questions on alcohol quantity, intake frequency and problematic drinking. Life events and traumatic experiences in childhood and adulthood were also asked about, as well as items on social interactions. We used a four-item life satisfaction scale and a brief assessment of extraversion and neuroticism, and also the Center for Epidemiologic Studies Depression (CES-D) depression scale.

Our analyses focused on four areas of interest: general health, health behaviours (viz., smoking habits, alcohol usage and coffee consumption), sleep issues and a broad sweep of psychosocial variables (viz., personality, depression, social interactions and history of traumatic experiences). The full questionnaire and items used in the analyses for the present study can be seen in detail at www. twinstudy.helsinki.fi. Ethical approval was obtained from the Ethics committee of the Helsinki and Uusimaa Hospital District. The study purpose was explained to the participants, and a returned questionnaire was taken as informed consent. All participants were aware they could withdraw from the study at any time without any consequence to themselves. 


\section{3 | STATISTICAL METHODS}

We used logistic regression analyses to explore the associations between putative explanatory factors and the frequency of $S B$ and $A B$ assessed using five-point questions as follows:

1. "Have you noticed or has someone told you (a person who sleeps with you) that you grind or clench your teeth while you're sleeping?" ( 1 = every night or almost every night, $2=3-5$ nights weekly, $3=1-2$ nights weekly, 4 = less than once a week, 5 = less than once a month or never).

2. "'Do you clench your teeth during wakefulness? ( 1 = every day or almost every day, 2 = 3-5 days weekly, 3 = 1-2 days weekly, $4=$ less than once a week, $5=$ less than once a month or never).

Four outcome variables were computed, two for SB and two for AB. We categorised and labelled them as (a) "any" sleep/awake bruxism (options 1-4) vs "rarely or none" (option 5), and (b) "frequent" sleep/awake bruxism (options 1 and 2) vs "rarely or none" (option 5). We standardised continuous variables to unit variances to enable comparisons of the strength of the associations across variables. The odds ratio thus provides an estimate of the change in odds per one standard deviation. Categorical variables were analysed using dummy binary variables, to give odds ratio estimates for each category relative to the chosen reference category.

Finally, we analysed twin similarity by computing polychoric correlations of the bruxism traits by zygosity and sex. Zygosity was determined by a validated questionnaire method. ${ }^{27}$ Also, to examine whether $A B$ and SB share a common genetic aetiology, we computed crosstwin-crosstrait correlations to determine the genetic correlation between them. This analysis allowed us to decompose the phenotypic correlation between $A B$ and $S B$ into genetic and environmental components and to examine to what extent shared genes accounted for the greater than expected co-occurrence between SB and $A B$ prevalence.

\section{4 | RESULTS}

Any SB was reported by $14.8 \%(n=1221)$ and frequent SB by $5.0 \%(n=412)$. Percentages for any and frequent $A B$ were $18.4 \%$ ( $n=1532)$ and $6.3 \%(n=522)$, respectively. Overall, both SB and $A B$ were slightly more often reported by women, without significant difference between the sexes. The proportion of participants reporting both $S B$ and $A B$ frequently was $29.5 \%$ among all persons with either frequent $S B$ or frequent $A B$ or both. The corresponding co-occurrence for any $A B$ and $S B$ within individuals was slightly less.

Bruxism behaviours were not overall associated with sleep duration. However, perceived poor sleep quality was positively associated with both $S B$ and $A B$, but showed higher $O R s$ for $A B$ than SB. Similarly, snoring, sleep apnoeas and insomnia were positively associated with both. However, the associations were stronger for sleep apnoeas with $S B$ than $A B$, while ORs were higher for $A B$ than
SB in relation to insomnia. In addition, a clear evening chronotype was positively associated with all studied bruxism. Odds ratios and their corresponding 95\% confidence intervals are shown in Table 1.

Several psychological variables related to mood (viz., depressive mood, neuroticism, negative life events and dissatisfaction with sex life) were positively associated with all studied SB and AB. Also, all various traumatic events reported were positively associated with reports of any SB and AB. These included serious accidents, either in childhood or in adulthood, violent crime in adulthood and sexual harassment. Parental divorce or separation showed an association with $A B$ but not $S B$. Likewise, those participants who were satisfied with life reported $S B$ and $A B$ significantly less often. Extraversion showed a protective association with $A B$ but no association with $S B$. Details are shown in Table 2.

Regarding social, lifestyle and health variables, we found that married participants reported $S B$ and $A B$ significantly less often, and those still in work life reported $A B$ less often. Alcohol consumption and related negative consequences (heavy drinking, passouts and hangovers) were positively associated with all studied SB and $A B$. For alcohol problems, the associations appeared stronger for $A B$ than SB. Similarly, smoking status and a measure of nicotine dependence were positively associated with both $S B$ and $A B$, but not coffee or tea use. Psychotropic medication use (viz., sedatives and antidepressants) was positively associated with all studied SB and $A B$. Poor subjective health was associated with $S B$ and somewhat more strongly with AB. Details are shown in Table 3.

Polychoric analyses examined twin similarity among $M Z$ and $D Z$ pairs for both $S B$ and $A B$. For $S B$, the intra-class correlation was 0.366 in $M Z$ pairs and 0.200 in DZ pairs, with very similar estimates in male and female pairs. The higher correlation in $M Z$ than in DZ pairs suggests the influence of additive genetic factors on SB. For $A B$, the $M Z$ correlation was 0.305 , and 0.083 in $D Z$ pairs, which is a finding consistent with the influence of both additive and non-additive genetic factors. However, given that $\mathrm{MZ}$ correlations were between 0.366 and 0.305 , environmental factors (including measurement error) accounted for around two-thirds of the variation in liability to SB and AB. In addition, we observed a crosstwin-crosstrait correlation among $M Z$ pairs twice as large as among DZ pairs, indicating a substantial genetic correlation between the two phenotypes (Table 4).

\section{5 | DISCUSSION}

Our main findings were the substantial correlates of behavioural and lifestyle factors with both $\mathrm{SB}$ and $\mathrm{AB}$, with fairly similar risk factor profiles. There was also substantial co-occurrence between the conditions, such that nearly one-third of persons reporting either condition had both. The role of genetic factors, based on twin correlations, was fairly similar but relatively modest for both $S B$ and $A B$. Also, SB and AB appear to share some genetic influences. This suggests that environmental factors play a major role in the aetiology of bruxism behaviours. 
TAB LE 1 Sleep and awake bruxism in relation to sleep related issues

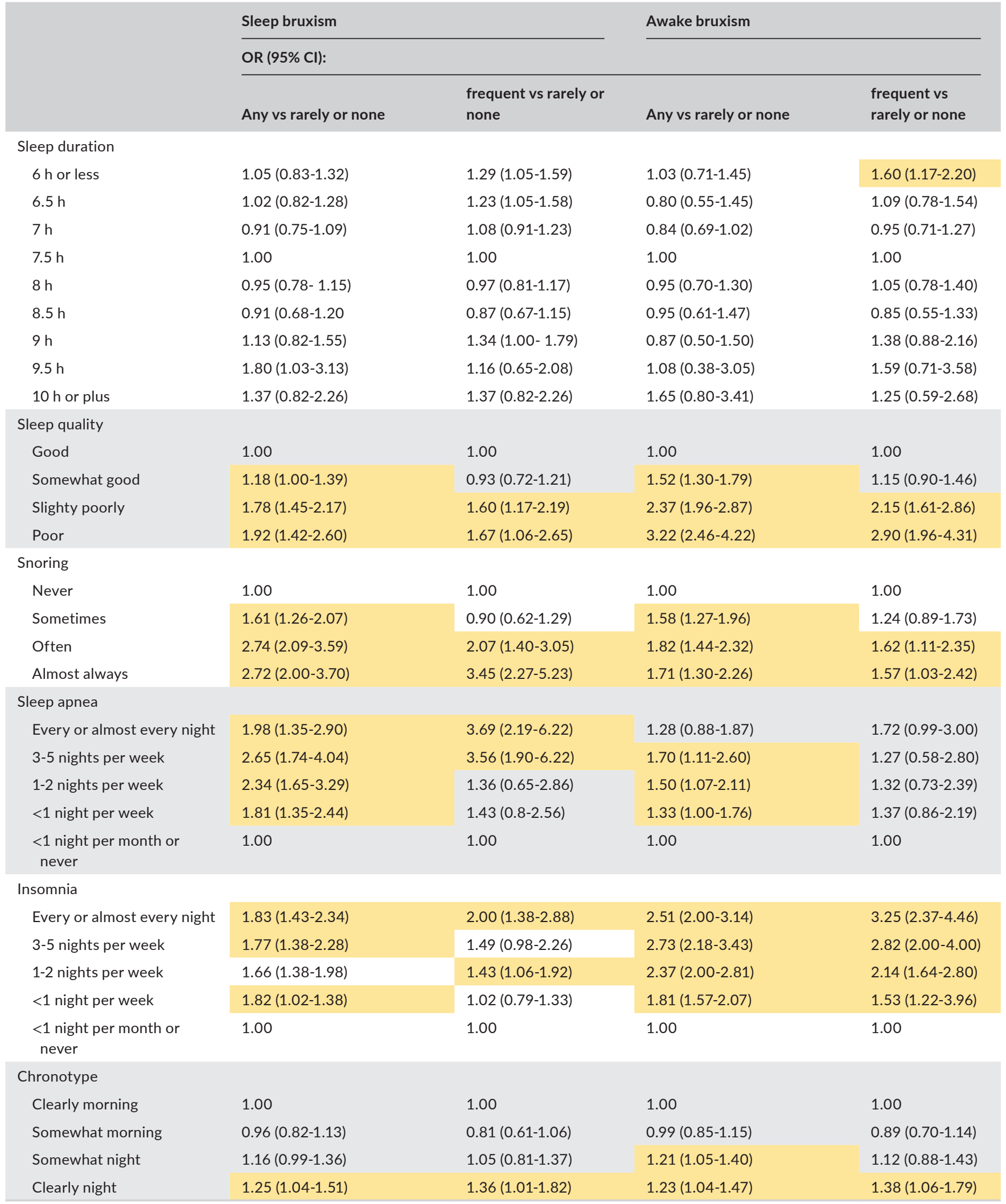

Note: Logistic regression, adjusted by age and sex. Odds ratios and their $95 \%$ confidence intervals. Complete frequency distributions are available from the authors upon request. 
TABLE 2 Sleep and awake bruxism in relation to psychological issues

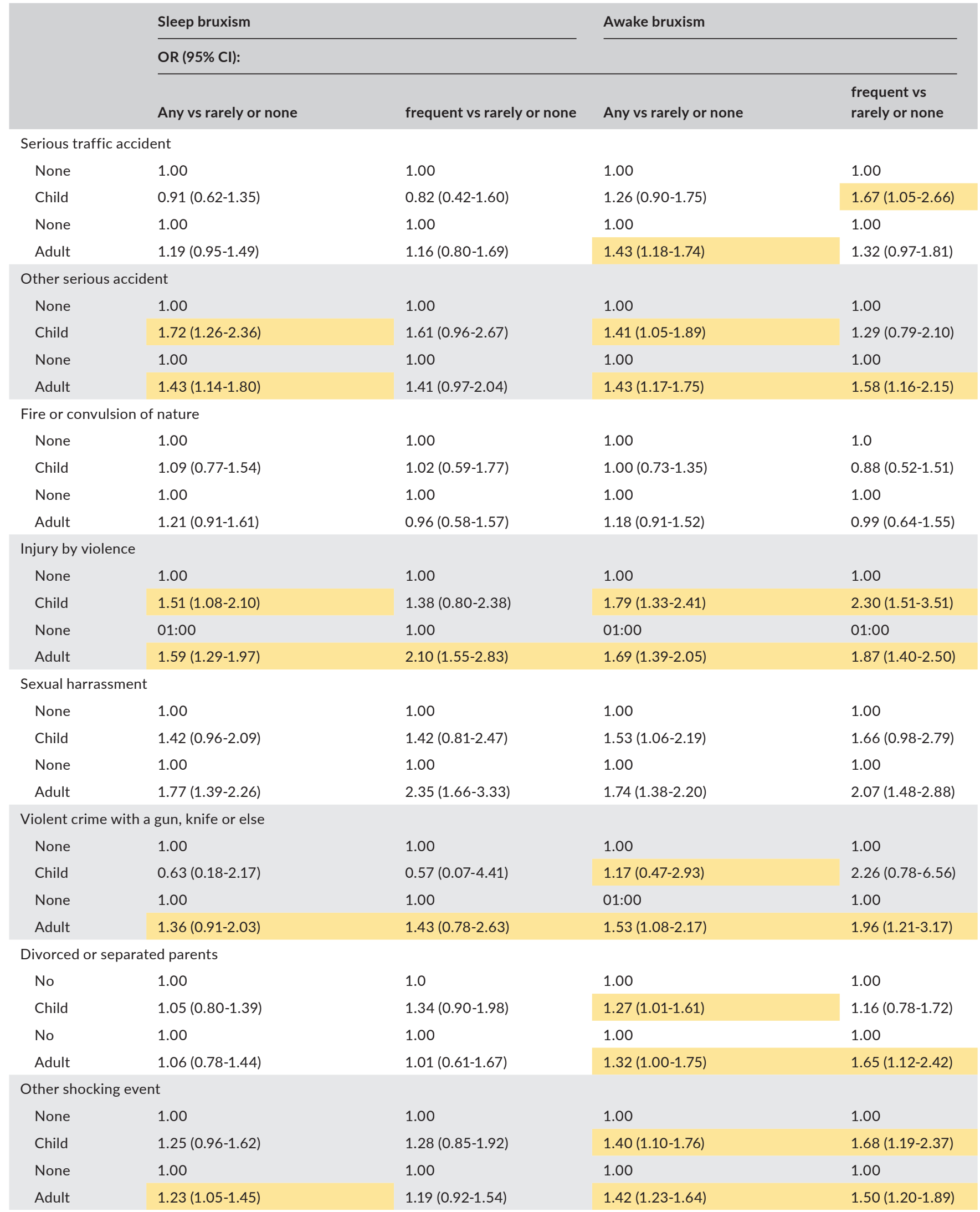


TABLE 2 (Continued)

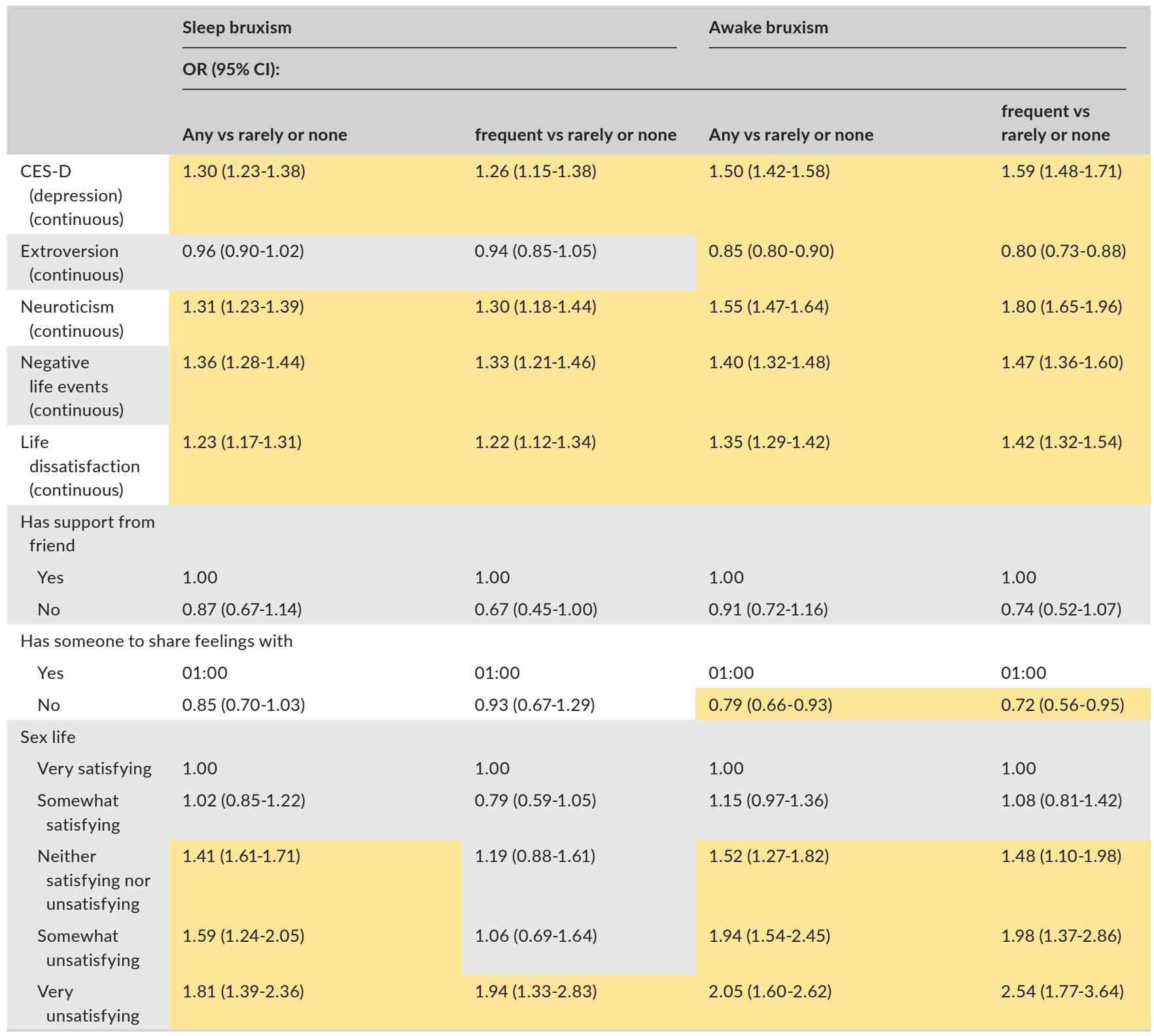

Note: Logistic regression, adjusted by age and sex. Odds ratios and their corresponding $95 \%$ confidence intervals (for continuous variables calculated by an additional standard deviation). Significant associations highlighted. Complete frequency distributions are available from the authors upon request.

Over two decades ago, Lavigne and Montplaisir $^{7}$ reported the prevalence of SB (at present considered as "possible" SB) to be about eight per cent, being at highest at 20-40 years, without significant difference between sexes, and declining with age. The commonly cited prevalence figures from this representative nationwide study among Canadians have been considered the most representative to date. According to a recent systematic review, $A B$ is reportedly more common than SB, with a prevalence of about $22 \%-30 \% .{ }^{20}$ However, the review's authors pointed out that the figures should be interpreted with caution due to the poor methodological quality of the reviewed literature. Their criticism mainly targeted the use of self-reported data. As with a vast number of diseases, it should be noted that the prevalence of both $\mathrm{SB}$ and $\mathrm{AB}$ (bearing in mind their non-disease entities by definition) may vary with place, time and characteristics of the population, including age, sex, genetic liability and external influences.

In the present study, based on questionnaire data derived from 8410 twins aged 53-67 years, the prevalences of frequent SB and any $A B$ were both in line with the previous literature. ${ }^{7,20}$ Bearing in mind that the figures imply "possible" bruxism, according to the international consensus, ${ }^{1}$ there is no other feasible method yet to gather data on bruxism behaviours on such a large scale other than using self-reports. Our study consisted of the nationwide Finnish twin cohort data with a comprehensive set of validated questions, and the study population is representative of the Finnish population at same age. ${ }^{26,28,29}$ 
TABLE 3 Sleep and awake bruxism in relation to health and lifestyle issues, marital status, work, and health. Logistic regression, adjusted by age and sex

\begin{tabular}{|c|c|c|c|c|}
\hline & \multicolumn{2}{|l|}{ Sleep bruxism } & \multicolumn{2}{|l|}{ Awake bruxism } \\
\hline & \multicolumn{4}{|l|}{ OR (95\% Cl): } \\
\hline $\begin{array}{l}\text { Alcohol consumption } \\
\text { (continuous) }\end{array}$ & $1.14(1.06-1.23)$ & $1.13(1.00-1.28)$ & $1.13(1.06-1.21)$ & $1.12(1.01-1.25)$ \\
\hline $\begin{array}{l}\text { Daily tea consumption } \\
\text { (continuous) }\end{array}$ & $0.94(0.87-1.01)$ & $0.89(0.79-1.00)$ & $1.01(0.95-1.08)$ & $1.00(0.90-1.10)$ \\
\hline $\begin{array}{l}\text { Heavy smoking index (HSI) } \\
\text { (continuous) }\end{array}$ & $1.18(1.09-1.29)$ & $1.23(1.07-1.41)$ & $1.20(1.11-1.30)$ & $1.26(1.13-1.42)$ \\
\hline Married again & $0.83(0.56-1.22)$ & $0.94(0.52-1.67)$ & $0.74(0.52-1.06)$ & $0.74(0.43-1.28)$ \\
\hline Cohabiting & $0.76(0.58-0.99)$ & $0.58(0.37-0.91)$ & $0.80(0.63-1.02)$ & $0.72(0.50-1.05)$ \\
\hline Divorced or separated & $1.08(0.86-1.36)$ & $1.09(0.76-1.55)$ & $1.01(0.81-1.25)$ & $1.09(0.79-1.49)$ \\
\hline Widowed & $0.89(0.63-1.25)$ & $0.76(0.43-1.33)$ & $0.80(0.58-1.10)$ & $0.72(0.44-1.19)$ \\
\hline \multicolumn{5}{|l|}{ Working } \\
\hline No & 1.00 & 1.00 & 1.00 & 1.00 \\
\hline Yes & $0.96(0.84-1.11)$ & $0.86(0.69-1.08)$ & $0.86(0.76-0.98)$ & $0.65(0.53-0.80)$ \\
\hline \multicolumn{5}{|l|}{ Subjective health } \\
\hline Very good & 1.00 & 1.00 & 1.00 & 1.00 \\
\hline No & 1.00 & 1.00 & 1.00 & 1.00 \\
\hline Yes & $1.20(0.96-1.51)$ & $1.36(0.96-1.94)$ & $1.35(1.10-1.66)$ & $1.48(1.08-2.02)$ \\
\hline \multicolumn{5}{|l|}{ Elevated blood pressure } \\
\hline No & 1.00 & 1.00 & 1.00 & 1.00 \\
\hline Yes & $1.09(0.97-1.24)$ & $1.02(0.83-1.25)$ & $1.16(1.04-1.30)$ & $1.26(1.05-1.51)$ \\
\hline \multicolumn{5}{|l|}{ Psychotropic medication use } \\
\hline \multicolumn{5}{|l|}{ Sedatives } \\
\hline No & 1.00 & 1.00 & 1.00 & 1.00 \\
\hline Yes & $1.69(1.45-1.96)$ & $1.82(1.43-2.33)$ & $1.85(1.61-2.11)$ & $2.40(1.96-2.94)$ \\
\hline \multicolumn{5}{|l|}{ Antidepressants } \\
\hline No & 1.00 & 1.00 & 1.00 & 1.00 \\
\hline Yes & $2.15(1.77-2.62)$ & $2.55(1.91-3.40)$ & $2.15(1.79-2.58)$ & $2.99(2.32-3.85)$ \\
\hline \multicolumn{5}{|l|}{ Smoking status } \\
\hline Never & 1.00 & 1.00 & 1.00 & 1.00 \\
\hline Occasionally & $1.14(0.87-1.50)$ & $1.01(0.64-1.60)$ & $1.37(1.08-1.74)$ & $1.60(1.08-2.36)$ \\
\hline Former & $1.27(1.09-1.48)$ & $1.38(1.09-1.76)$ & $1.39(1.21-1.60)$ & $1.90(1.52-2.38)$ \\
\hline Current & $1.69(1.43-2.00)$ & $1.60(1.22-2.11)$ & $1.70(1.45-1.99)$ & $2.42(1.89-3.11)$ \\
\hline
\end{tabular}


TABLE 3 (Continued)

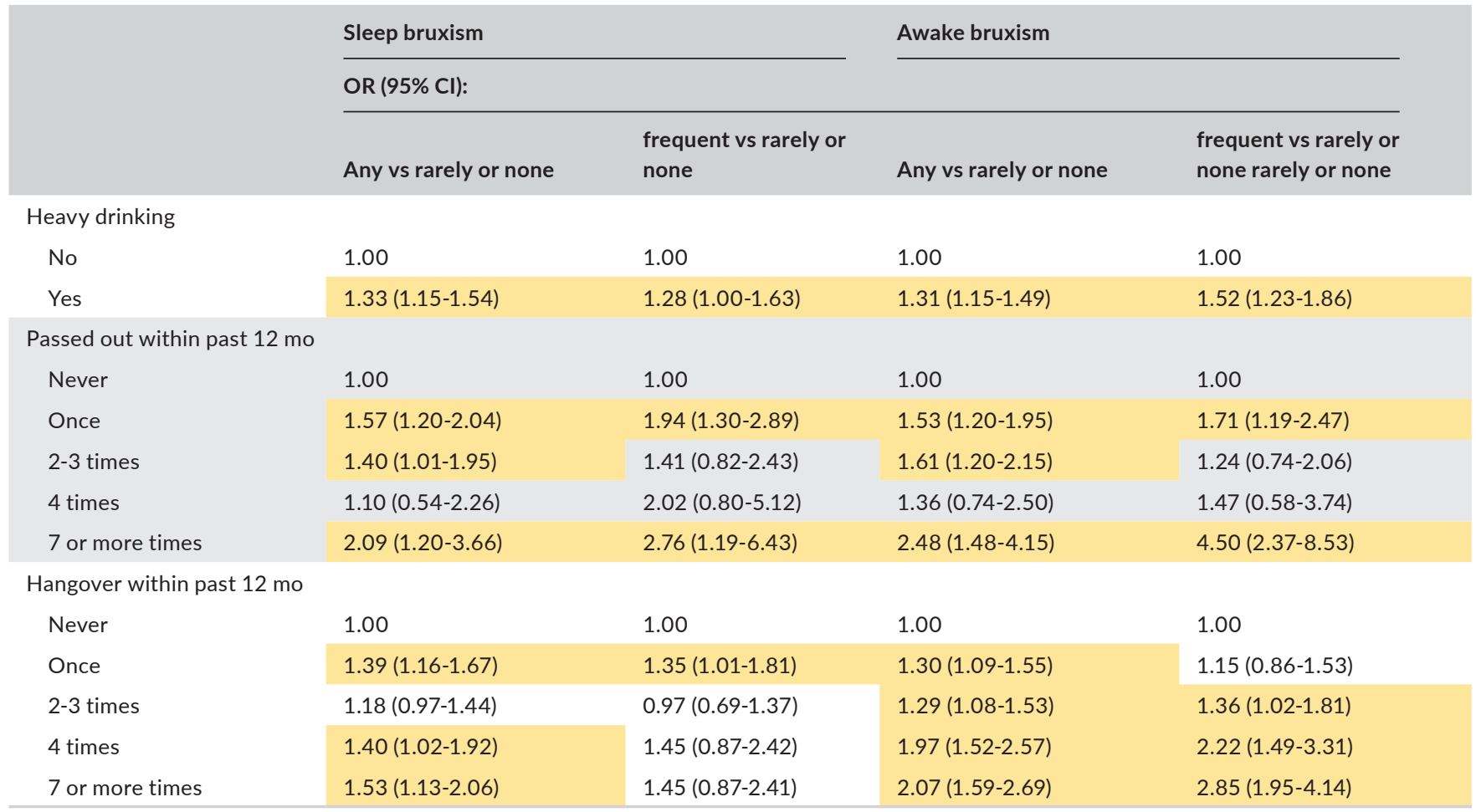

Odds ratios and their $95 \%$ confidence intervals (for continuous variables calculated by an additional standard deviation). Significant associations highlighted. Complete frequency distributions are available from the authors upon request.

TABLE 4 Intra-class and crosstwincrosstrait correlations for sleep and awake bruxism by zygosity and sex

\begin{tabular}{lllll}
\hline Trait & Zygosity & $\begin{array}{l}\text { All pairs } \\
(\mathrm{N}=2771)\end{array}$ & $\begin{array}{l}\text { Male } \\
(\mathrm{N}=1123)\end{array}$ & $\begin{array}{l}\text { Female } \\
(\mathrm{N}=1648)\end{array}$ \\
\hline Sleep bruxism & $\mathrm{MZ}(\mathrm{n}=1009)$ & 0.366 & 0.362 & 0.373 \\
\hline Awake bruxism & $\mathrm{DZ}(\mathrm{n}=1775)$ & 0.200 & 0.214 & 0.284 \\
\hline $\begin{array}{c}\text { Crosstwin-crosstrait } \\
\text { correlations }\end{array}$ & $\mathrm{MZ}(\mathrm{n}=1020)$ & 0.305 & 0.277 & 0.328 \\
\hline & $\mathrm{DZ}(\mathrm{n}=1795)$ & 0.083 & 0.107 & 0.068 \\
\hline
\end{tabular}

Note: Column numbers are for pairs in the crosstwin-crosstrait correlations. For sleep and awake bruxism intra-class correlations, the numbers are slightly higher due to some pairs with missing data on one trait but not the other.
$S B$ and $A B$ have usually been considered as different entities without mutual pathophysiology or physiopathology of the bruxism behaviours, not to mention the origin of these oromotor muscle activities in the brain. ${ }^{1,5}$ However, our findings demonstrate that both behaviours share quite similar correlate profiles. For example, perceived sleep quality was significantly associated with both SB and $A B$ (any vs. never or less than a month). However, sleep apnoeas were not associated with any $A B$, which has not been reported earlier. A closer relationship between SB and apnoeas may indicate a possible role of SB in enhancing airway patency during sleep arousals or comorbidity. ${ }^{17,18}$

The master biological clock, located in the bilaterally paired suprachiasmatic nucleus of the anterior hypothalamus, controls the timing of sleep and waking in humans and regulates the 24-hour (ie circadian) behavioural, physiologic and biologic rhythms. ${ }^{30}$ There are two extremes in the so-called chronotype profiles: morningness (individuals who wake up early, are at their most active early, and go to sleep early) and eveningness (individuals who wake up late, are most active later, and go to sleep late). These two profiles may not always be balanced with social activities and work duties, for example. In addition, several psychological factors may be associated with chronotype profiles. ${ }^{31}$ Nevertheless, chronotype profiles reportedly become less distinct by age and morningness becomes more common with increasing age among adults. ${ }^{32}$ However, in the present study among twins aged 53-67 years, the evening type still seemed to associate with both studied bruxism behaviours. 
It is noteworthy that a wide array of psychosocial measures (viz., depression, neuroticism and negative life events) was significantly associated with all studied bruxism behaviours. This is in line with previous findings. ${ }^{12,14}$ That the use of psychotropic medicines was significantly associated with all bruxism behaviour also accords with existing literature. ${ }^{15,16}$ Interestingly, serious traumatic events both in adulthood and childhood were associated with bruxism behaviours overall. This should be noted; such events may become stored in the amygdala, the limbic system of the brain, and may have a life-long influence on bruxism behaviour too-an issue that needs to be borne in mind as a possible underlying factor when encountering a patient with a possible bruxism-related problem.

Furthermore, the results confirm previously reported significant associations between SB and smoking, and alcohol consumption. ${ }^{16}$ In the present study, heavy drinkers, especially those with alcohol-related problems, also seemed to be at risk of more severe $A B$. All of which should be taken into account during a patient visit in terms of any treatment planning.

The present study summarised results from numerous age and sex adjusted logistic regression models. Corresponding large scale findings have not been reported earlier. Despite the models not being corrected for multiple testing, the reader is asked to focus on the strength of the found associations. It should also be noted that multiple correlates for both $\mathrm{SB}$ and $\mathrm{AB}$ were found to be very similar, but we also saw some non-shared items between studied variables and bruxism behaviours.

Interestingly, a substantial co-occurrence between $\mathrm{SB}$ and $\mathrm{AB}$ emerged, which raises the question of whether they also share a common genetic liability. The twin analyses suggested moderate genetic effects on both $S B$ and $A B$ and that there is a substantial genetic correlation between the two phenotypes.

Notwithstanding the evidence for genetic liability, external factors appear to play a major role in the aetiology of bruxism. However, it should be noted that the aetiology of bruxism could be unrelated to clinical consequences, ${ }^{33}$ and moreover that other constructs (viz., risk or trigger factors to bruxism) should be borne in mind when a patient complains of symptoms that could be linked in involuntary masticatory muscle work, viz., bruxism.

In conclusion, SB and AB may be two phenotypes with similar origin. In addition to shared genetics, both are markedly associated with several risk factors. Some of the negative outcomes of bruxism behaviours related to the latter could perhaps be reduced by lifestyle changes. Psychological characteristics and a history of traumatic events should be taken into account in clinical work when facing a patient with a possible bruxism-related problem.

\section{ACKNOWLEDGMENTS}

The authors of this manuscript are grateful to Richard K. Burton, B.Sc., Director, Irish Institute for Nutrition and Health, Dublin, Ireland, for the careful language editing and revisions. The tables were carefully edited by Aino Ahlberg, M.Sc., Faculty of Medicine, University of Helsinki, Finland.

\section{CONFLICTS OF INTEREST}

The Dr Lobbezoo reports grants and other from Sunstar Suisse SA, grants from Somnomed-Goedegebuure, grants from Airway Management, all outside the submitted work. All other authors report no conflicts of interest concerning this manuscript.

\section{AUTHORS' CONTRIBUTIONS}

$\mathrm{JA}$ and JK drafted the manuscript on the basis of the statistical the analyses by JK. All other expert authors critically revised the text and made comments on the manuscript.

\section{PEER REVIEW}

The peer review history for this article is available at https://publo ns.com/publon/10.1111/joor.13042.

\section{ORCID}

Jari Ahlberg (iD https://orcid.org/0000-0002-6052-0441

Frank Lobbezoo iD https://orcid.org/0000-0001-9877-7640

Daniele Manfredini iD https://orcid.org/0000-0002-4352-3085

Ghizlane Aarab (iD https://orcid.org/0000-0002-6677-7897

\section{REFERENCES}

1. Lobbezoo F, Ahlberg J, Glaros A, et al. Bruxism defined and graded: an international consensus. J Oral Rehabil. 2013;40:2-4.

2. De Leeuw R, Klasser GD. Orofacial pain. Guidelines for assessment, diagnosis, and management, 5th ed. Chicago, IL: Quintessence Publishing Co Inc; 2013.

3. De Leeuw R, Klasser GD. Orofacial pain. Guidelines for assessment, diagnosis, and management, 6th ed. Chicago, IL: Quintessence Publishing Co Inc; 2018.

4. American Academy of Sleep Medicine. International classification of sleep disorders, 3rd ed. Westchester, NY: Academy American of Sleep Medicine; 2014.

5. Lobbezoo F, Ahlberg J, Raphael K, et al. International consensus on the assessment of bruxism: report of a work in progress. J Oral Rehabil. 2018;00:1-8.

6. Mandfredini D, Ahlberg J, Wetselaar P, Svensson P, Lobbezoo F. The bruxism construct: from cut-off points to a continuum spectrum. $J$ Oral Rehabil. 2019;46:991-997.

7. Lavigne G, Montplaisir J. Restless legs syndrome and sleep bruxism: prevalence and associations among Canadians. Sleep. 1994;17:739-743.

8. Ohayon M, Li K, Guilleminault C. Risk factors for sleep bruxism in the general population. Chest. 2001;119:53-61.

9. Hublin C, Kaprio J, Partinen M, Koskenvuo M. Sleep bruxism based on self-report in a nationwide twin cohort. J Sleep Res. 1998;7:61-67.

10. Rintakoski K, Hublin C, Lobbezoo F, Rose RJ, Kaprio J. Genetic factors account for half of the phenotypic variance in liability to sleep-related bruxism in young adults: a nationwide Finnish twin cohort study. Twin Res Hum Genet. 2012;15:714-719.

11. Lobbezoo F, Visscher CM, Ahlberg J, Manfredini D. Bruxism and genetics: a review of the literature. J Oral Rehabil. 2014;41:709-714.

12. Ahlberg J, Lobbezoo F, Ahlberg K, et al. Self-reported bruxism mirrors anxiety and stress in adults. Med Oral Patol Oral Cir Bucal. 2013:18:e7-e11.

13. Manfredini D, Fabbri A, Peretta R, Guarda-nardini L, Lobbezoo F Influence of psychological symptoms on home-recorded sleeptime masticatory muscle activity in healthy subjects. J Oral Rehabil. 2011;38:902-911 
14. Manfredini D, Lobbezoo F. Role of psychosocial factors in the etiology of bruxism. J Orofac Pain. 2009;23:153-166.

15. Lobbezoo F, van Denderen RJ, Verheil JG, Naeije M. Reports of SSRI-related bruxism in the family physicians office. J Orofac Pain. 2001;15:340-346.

16. Rintakoski K, Kaprio J. Legal psychoactive substances as risk factors for sleep-related bruxism: a Nationwide Finnish Twin Cohort Study. Alcohol Alcohol. 2013;48:487-494.

17. Manfredini D, Guarda-Nardini L, Marchese-Ragona R, Lobbezoo F. Theories on possible temporal relationships between sleep bruxism and obstructive sleep apnea events. An expert opinion. Sleep Breath. 2015;19:1459-1465.

18. Hesselbacher S, Subramanian S, Rao S, Casturi L, Surani S. Selfreported sleep bruxism and nocturnal gastroesophageal reflux disease in patients with sleep apnea: relationship to gender and ethnicity. Open Respir Med. 2014;8:34-40.

19. Manfredini D, Ahlberg J, Winocur E, Lobbezoo F. Management of sleep bruxism in adults: a qualitative systematic literature review. J Oral Rehabil. 2015;42:862-874.

20. Manfredini D, Winocur E, Guarda-Nardini L, Paesani D, Lobbezoo F. Epidemiology of bruxism in adults: a systematic review of the literature. J Orofac Pain. 2013;27:99-110.

21. Van der Zaag J, Lobbezoo F, Visscher CM, Hamburger HL, Naeije $M$. Time-variant nature of sleep bruxism outcome variables using ambulatory polysomnography: implications for recognition and therapy evaluation. J Oral Rehabil. 2008;35:577-584.

22. Bracci A, Djukic G, Favero L, Salmaso L, Guarda-Nardini L, Manfredini D. Frequency of awake bruxism behaviours in the natural environment. A 7-day, multiple-point observation of real-time report in healthy young adults. J Oral Rehabil. 201845(6):423-429.

23. Zani A, Lobbezoo F, Bracci A, Ahlberg J, Manfredini D. Ecological momentary assessment and intervention principles for the study of awake bruxism behaviors, Part 1: general principles and preliminary data on healthy young italian adults. Front Neurol. 2019;10:169. https://doi.org/10.3389/fneur.2019.00169

24. Osiewicz MA, Lobbezoo F, Bracci A, Ahlberg J, Pytko-Polończyk J, Manfredini D. Ecological momentary assessment and intervention principles for the study of awake bruxism behaviors, Part 2: development of a smartphone application for a multicenter investigation and chronological translation for the polish version. Front Neurol. 2019;10:170. https://doi.org/10.3389/fneur.2019.00170

25. Lavigne G, Khoury S, Abe S, Yamaguchi T, Raphael K. Bruxism physiology and pathology: an overview for clinicians. J Oral Rehabil. 2008;35:476-494.

26. Kaprio J, Bollepalli S, Buchwald J, et al. The older finnish twin cohort - 45 years of follow-up. Twin Res Hum Genet. 2019;22:240-254.

27. Sarna S, Kaprio J, Sistonen P, Koskenvuo M. Diagnosis of twin zygosity by mailed questionnaire. Hum Hered. 1978;28:241-254.

28. Kaprio J. The Finnish Twin Cohort Study: an update. Twin Res Hum Genet. 2013;16:157-162.

29. Skytthe A, Harris JR, Czene K, et al. Cancer incidence and mortality in 260000 Nordic twins with prospective cancers. Twin Res Hum Genet. 2019;22:99-107.

30. Turek F, Dugovic C, Laposky A. Master circadian clock, master circadian rhythm. In: Kryger MH, Roth T, Dement WC, eds. Principles and practice sleep medicine. Philadelphia, PA: Elsevier Saunders; 2005:318-320.

31. Simon P, Zavecz Z, Pálosi V, Török C, Köteles F. The influence of sleep complaints on the association between chronotype and negative emotionality in young adults. Chronobiol Int. 2015;32:1-10.

32. Broms U, Pitkäniemi J, Bäckman $\mathrm{H}$, et al. Long-term consistence of diurnal-type preferences among men. Chronobiol Int. 2014;3:182-188.

33. Manfredini D, De Laat A, Winocur E, Ahlberg J. Why not stop looking at bruxism as a black/white condition? Etiology could be unrelated to clinical consequences. J Oral Rehabil. 2016;43:799-801.

How to cite this article: Ahlberg J, Piirtola M, Lobbezoo F, et al. Correlates and genetics of self-reported sleep and awake bruxism in a nationwide twin cohort. J Oral Rehabil. 2020;47:1110-1119. https://doi.org/10.1111/joor.13042 